\title{
Treatment of alcohol dependence
}

\begin{abstract}
It is important to manage alcohol withdrawal properly. Mismanagement could lead to permanent brain damage or even death. The principles are simple and easily learnt. New pharmacological agents have come into use which helps in maintaining abstinence. Clinicians should update themselves on their use.
\end{abstract}

\section{Introduction}

The symptoms of alcohol withdrawal can range from mild to life threatening. Though withdrawal is best managed in an inpatient unit, suitable patients could be managed on an outpatient basis [1].

\section{Panel 1. Relative indications for inpatient alcohol detoxification [1]}

- History of severe withdrawal symptoms

- Multiple past detoxifications

- History of alcohol withdrawal seizures or delirium tremens

- Concomitant medical or psychiatric illness

- Recent high levels of alcohol consumption

- Lack of reliable support network

- Pregnancy

The symptoms of alcohol withdrawal are variable in type and severity. They occur within hours of the last drink and peak within 24-48 hours.

\section{Panel 2. Symptoms and signs of acute alcohol withdrawal [2]}

Anxiety, agitation and insomnia

Tachycardia and sweating

Tremor of limbs, tongue and eyelids

Nausea and vomiting

Seizures

Confusion and hallucinations

In most individuals the symptoms of withdrawal are mild to moderate and do not last beyond a week. In about $5 \%$ it could lead to delirium tremens (DTs). DTs present insidiously with nocturnal confusion. If not treated promptly it carries a mortality of $1-2 \%$ [3].

\section{Panel 3. Symptoms and signs of delirium tremens [3]}

Confusion and disorientation

Agitation

Tachycardia and hypertension

Fever

Visual and auditory hallucinations

Paranoid ideation

\section{Pharmacological treatment of alcohol withdrawal}

Drug treatment is most useful in moderate to severe withdrawal. Many drugs have been used but mainstay of therapy is the use of a benzodiazepine. A withdrawal scale is useful in assessing severity of withdrawal. This minimises over or under dosing. The Clinical Institute Alcohol Withdrawal Assessment Scale, Revised (CIWA-Ar) is a ten item scale which can be completed in five minutes [4]. A long acting benzodiazepine such as chlordiazepoxide or diazepam is more effective in preventing seizures and DTs but a short acting benzodiazepine such as lorazepam or oxazepam is safer in those with liver failure.

\section{Use of benzodiazepines}

Benzodiazepines could be given in several different ways. In a flexible dose regime the dose is determined according to the symptoms. This is suitable in a unit where the nursing staff are trained to detect withdrawal symptoms. A fixed interval tapering dose regime is more practical in a general ward regime.

A regime suitable for an individual with moderate alcohol dependence is given below. A person with severe alcohol dependence would need higher doses (for e.g. 40 $\mathrm{mg}$ qds on day 1) and is best managed in a specialised unit. A stat dose of chlordiazepoxide $40 \mathrm{mg}$ to $80 \mathrm{mg}$ would be useful in such people. Chlordiazepoxide may be discontinued in the majority after one week.

Panel 4. Chlordiazepoxide regime for an individual with moderate alcohol dependence

$\begin{array}{ll}\text { Day } 1 & 20 \mathrm{mg} \mathrm{qds} \\ \text { Day } 2 & 15 \mathrm{mg} \mathrm{qds} \\ \text { Day } 3 & 10 \mathrm{mg} \mathrm{qds} \\ \text { Day } 4 & 5 \mathrm{mg} \mathrm{qds} \\ \text { Day } 5 & 5 \mathrm{mg} \mathrm{bd}\end{array}$




\section{Vitamin supplementation}

Give adequate doses of thiamine to all those undergoing inpatient detoxification to prevent the onset of Wernicke's encephalopathy (WE). WE is described as presenting with the classic triad of opthalmoplegia, ataxia and confusion. All three signs are rarely present in clinical practice. Suspect all patients undergoing alcohol detoxification who present with ataxia, hypothermia, hypotension, confusion, opthalmoplegia, nystagmus, memory disturbances, or impaired level of consciousness of having WE. Give a minimum of $100 \mathrm{mg}$ of thiamine IM daily for 5 days. If pure thiamine is not available use a proprietary preparation containing adequate amounts of thiamine. Oral thiamine is unlikely to be absorbed adequately. Administer thiamine before parenteral glucose as this may precipitate thiamine deficiency. Inadequate or non administration of thiamine may lead to Korsakov's syndrome characterised by devastating short term memory loss. In some the damage is permanent.

\section{Other medication and measures}

Use of anticonvulsants for seizure prophylaxis is not necessary as the benzodiazepines provide adequate cover. If a patient has prominent hallucinations use haloperidol, keeping in mind that it could reduce the seizure threshold and cause hypotension and prolongation of the QT interval. Give adequate fluid to maintain hydration and electrolyte balance.

\section{Pharmacotherapy for relapse prevention}

Pharmacological therapy has been found to be most effective when combined with some form of specialised counselling [5]. Four drugs have been found to be useful in the prevention of relapse.

\section{Disulfiram}

This is the oldest. Disulfiram inhibits aldehyde dehydrogenase leading to the accumulation of acetaldehyde when taken with alcohol leading to unpleasant and, with larger amounts, dangerous physical effects such as arrhythmias and hypotension. Make the person fully aware of the consequences before starting on the drug. A dose of $250 \mathrm{mg}$ daily given in the morning is adequate. Though liver disease is given as a contraindication most people who would benefit from it would have some degree of impairment. The use of disulfiram in those with moderate elevation of liver enzymes without liver failure should be safe. Disulfiram is suitable for motivated individuals who need additional support to prevent them taking alcohol when faced with social and environmental cues. The effectiveness of disulfiram is increased if such a person agrees to take it under supervision. It is usually given for a period of 3 to 6 months after which preventive behavioural strategies are established.

\section{Acamprosate}

Acamprosate given in a dose of $666 \mathrm{mg}$ t.d.s (body weight over $60 \mathrm{~kg}$ ) has been found to be effective in maintaining abstinence once a person has stopped drinking for over a week [6]. Heavy drinking leads to abnormality of the glutamate system, craving being one symptom. Acamprosate is thought to act by normalising the brain glutamate system. It works best in people who remain abstinent during treatment though concomitant use does not cause a reaction unlike with disulfiram. Acamprosate should be given for 6 months or more. The thrice a day dose however might lead to significant compliance problems.

\section{Naltrexone}

Naltrexone is an opioid antagonist. It blocks the release of endogenous endorphins which are thought to be released on consumption of alcohol leading to positive reinforcement effects. Given in a dose of 50mg (one tab) daily it has been shown to significantly reduce the rate of relapse as well as heavy drinking days [7]. The once daily dose is an added advantage.

\section{Topiramate}

Topiramate is currently used in the treatment of epilepsy and migraine. Though not yet approved by the FDA for relapse prevention, recent studies have demonstrated the usefulness of topiramate in alcohol dependence [8]. Another more recent study has shown topiramate to be useful in improving physical functioning (reduction of blood pressure, lowering cholesterol levels and decreased liver enzymes) as well as reducing obsessional thoughts and compulsions regarding alcohol use [9]. Doses up to $300 \mathrm{mg}$ per day were used but many responded to lower doses. It is important to titrate the drug gradually to minimise side-effects.

\section{Psychosocial intervention}

Three psychosocial strategies are commonly employed. They are cognitive behavioural therapy (CBT), motivational enhancement therapy (MET) and twelve-step facilitation (TSF). Cognitive behavioural therapy (CBT) is aimed at dealing with thoughts about alcohol, urges, refusing drinks, and avoiding situations that might lead to relapse. Motivational enhancement therapy (MET) based on principles of motivational psychology focuses on producing internally motivated change. Twelve-step facilitation (TSF) is based on the principles of AA. Project MATCH a large, multicenter trial designed to match the most effective psychosocial treatment to individual patient characteristic found all three therapies to be equally effective [10]. 


\section{Further Reading}

1. Gelder MG, Cowen P, Harrison PJ. Shorter Oxford Textbook of Psychiatry. 5th ed. Oxford: Oxford University Press; 2006.

2. Taylor D, Paton C, Kerwin R, South London and Maudsley NHS Trust, Oxleas NHS Foundation Trust. Prescribing Guidelines. 9th ed. Taylor \& Francis; 2007.

\section{References}

1. Myrick H, Anton RF. Treatment of alcohol withdrawal. Alcohol Health and Research World 1998; 22: 38-43.

2. Ritson B. Treatment for alcohol related problems. British Medical Journal 2005; 330: 139-41.

3. Taylor DM, The South London and Maudsley NHS Trust Prescribing Guidelines. 9th ed. 2007, London: Informa Healthcare.

4. Sullivan JT, Sykora K, Schneiderman J, Naranjo CA, Sellers EM. Assessment of alcohol withdrawal: the revised clinical institute withdrawal assessment for alcohol scale (CIWA-Ar). British Journal of Addiction 1989; 84: 1353-7.

5. Anton RF, O'Malley SS, Ciraulo DA, Cisler RA, Couper D, Donovan DM, et al. Combined pharmacotherapies and behavioural interventions for alcohol dependence: the COMBINE study: a randomized controlled trial. Journal of the American Medical Association 2006; 295: 2003-17.

6. Mann K, Lehert P, Morgan MY. The efficacy of acamprosate in the maintenance of abstinence in alcohol-dependent individuals: results of a meta-analysis. Alcoholism: Clinical and Experimental Research 2004; 28: 51-63.

7. Bouza C, Angeles M, Munoz A, Amate JM. Efficacy and safety of naltrexone and acamprosate in the treatment of alcohol dependence: a systematic review. Addiction 2004; 99: $811-28$

8. Johnson BA, Rosenthal N, Capece JA, Wiegand F, Mao L, Beyers K, et al. Topiramate for treating alcohol dependence: a randomized controlled trial. Journal of the American Medical Association 2007; 298: 1641-51.

9. Johnson BA, Rosenthal N, Capece JA, Wiegand F, Mao L, Beyers K, et al. Improvement of physical health and quality of life of alcohol-dependent individuals with topiramate treatment: US multisite randomized controlled trial. Archives of Internal Medicine 2008; 168: 1188-99.

10. Matching alcoholism treatments to client heterogeneity: project MATCH post-treatment drinking outcomes. Journal of Studies on Alcohol and Drugs 1997 58: 7-29.

Raveen Hanwella and Varuni de Silva, Department of Psychological Medicine, Faculty of Medicine, Colombo, Sri Lanka.

Correspondence: RH, e-mail <raveenhanwella@yahoo.co.uk>. Competing interests: none declared. 